\title{
On-surface synthesis of graphene nanoribbons with zigzag edge topology
}

Pascal Ruffieux ${ }^{1 *}$, Shiyong Wang ${ }^{1 *}$, Bo Yang ${ }^{2 *}$, Carlos Sánchez-Sánchez ${ }^{1 *}$, Jia Liu $^{1 *}$, Thomas Dienel ${ }^{1}$, Leopold Talirz ${ }^{1}$, Prashant Shinde ${ }^{1}$, Carlo A. Pignedoli ${ }^{1,3}$, Daniele Passerone ${ }^{1}$, Tim Dumslaff ${ }^{2}$, Xinliang Feng ${ }^{4}$, Klaus Müllen ${ }^{2}$ \& Roman Fasel ${ }^{1,5}$

\begin{abstract}
Graphene-based nanostructures exhibit electronic properties that are not present in extended graphene. For example, quantum confinement in carbon nanotubes and armchair graphene nanoribbons leads to the opening of substantial electronic bandgaps that are directly linked to their structural boundary conditions $\mathbf{s}^{1,2}$. Nanostructures with zigzag edges are expected to host spinpolarized electronic edge states and can thus serve as key elements for graphene-based spintronics ${ }^{3}$. The edge states of zigzag graphene nanoribbons (ZGNRs) are predicted to couple ferromagnetically along the edge and antiferromagnetically between the edges ${ }^{4}$, but direct observation of spin-polarized edge states for zigzag edge topologies-including ZGNRs—has not yet been achieved owing to the limited precision of current top-down approaches ${ }^{5-10}$. Here we describe the bottom-up synthesis of ZGNRs through surfaceassisted polymerization and cyclodehydrogenation of specifically designed precursor monomers to yield atomically precise zigzag edges. Using scanning tunnelling spectroscopy we show the existence of edge-localized states with large energy splittings. We expect that the availability of ZGNRs will enable the characterization of their predicted spin-related properties, such as spin confinement ${ }^{11}$ and filtering ${ }^{12,13}$, and will ultimately add the spin degree of freedom to graphene-based circuitry.
\end{abstract}

Graphene nanoribbons with armchair edges (AGNRs) can be synthesized using a bottom-up approach based on surface-assisted polymerization and subsequent cyclodehydrogenation of oligophenylene precursor monomers ${ }^{14}$. On-surface synthesis has been applied to fabricate many different AGNR structures ${ }^{14-16}$, N-doped AGNRs ${ }^{17,18}$ as well as AGNR heterostructures ${ }^{18,19}$. However, it is not directly suited for ZGNRs because polymerization of monomers via aryl-aryl coupling takes place along the armchair direction rather than the zigzag direction (Fig. 1a). Additionally, dehydrogenative cyclization of phenyl subgroups is not sufficient to form pure zigzag edges. Therefore, additional carbon functions must be placed at the edges of the monomers to complete the tiling toolbox needed for the bottom-up fabrication of arbitrary GNR structures.

In our protocol, surface-assisted polymerization and subsequent cyclization of suitably designed molecular precursors enables the synthesis of ZGNRs with control over both ribbon width and edge morphology. The idea depends on the choice of a U-shaped monomer (1), shown in Fig. 1b. With two halogen functions for thermally induced aryl-aryl coupling at the $\mathrm{R}_{1}$ positions, this monomer allows surfaceassisted polymerization to make a snake-like polymer. This monomer design can, in principle, be generalized to form wider structures by adding additional phenyl groups at the $\mathrm{R}_{1}$ position. Furthermore, it affords accommodation of additional phenyl groups at the $\mathrm{R}_{2}$ position that fill the holes in the interior of the undulating polymer. The crucial precursor is monomer 1a, which carries two additional methyl groups. In this case, as well as the polymerization and planarization, an oxidative ring closure including the methyl groups is expected that would then establish two new six-membered rings together with the zigzag edge structure. The choice of $R_{3}$ further enables either the growth of pure zigzag edges $\left(\mathrm{R}_{3}=\mathrm{H}, \mathbf{1 a}\right)$ or the addition of further functional groups to the edges $(\mathbf{1 b})$.

Monomer 1a was successfully obtained via multi-step organic synthesis (see Supplementary Information) and deposited on the clean $\mathrm{Au}(111)$ single-crystal surface by thermal sublimation under ultra-high vacuum conditions. If the surface is held at the dehalogenation temperature of $475 \mathrm{~K}$, then precursor monomers are immediately activated and undergo polymer formation via radical addition (step 1 in Fig. 1c). Further annealing to the cyclodehydrogenation temperature of $625 \mathrm{~K}$ is then applied to form the final 6-ZGNR (that is, a ZGNR that is six carbon zigzag lines wide; step 2 in Fig. 1c). This two-step process has been successfully monitored by scanning tunnelling microscopy (STM; Fig. 2a, b). Large-scale STM images of the Au(111) surface after deposition of precursor monomer la onto the substrate held at a temperature of $475 \mathrm{~K}$ reveal the formation of long (approximately $50 \mathrm{~nm}$ ) polymers for which the meandering maxima in the apparent height have a periodicity of $1.55 \mathrm{~nm}$, evidencing covalent-bond formation between the precursor monomers (Fig. 1c). The maxima with apparent heights of $0.3 \mathrm{~nm}$ are attributed to the sterically induced out-of-plane conformation of the phenyl ring carrying the methyl groups. Further annealing the sample to $625 \mathrm{~K}$ results in a complete planarization of the linear structures and a decrease in apparent height to $0.2 \mathrm{~nm}$, consistent with the formation of the fully conjugated ribbon structure ${ }^{14}$. Small-scale images (inset of Fig. 2b) reveal completely smooth and flat edge areas. This indicates that, in addition to the cyclodehydrogenation of the two phenyl rings, the methyl groups are dehydrogenatively incorporated to form a fully conjugated system with atomically precise zigzag edges. Further structural details are accessible by non-contact atomic force microscopy (nc-AFM) imaging with a CO-functionalized tip (Fig. 2c), which enables direct imaging of the local bond configurations at small distances ${ }^{20}$. The achieved resolution directly confirms that the observed width and edge morphology correspond to the expected $6-Z G N R$ structure as defined by the design of 1a. Furthermore, we can unambiguously state that the zigzag-edge atoms have the expected mono-hydrogen termination. Other possible terminations such as radical edges due to complete dehydrogenation or $\mathrm{H}_{2}$ termination can be disregarded, owing to the absence of bending across the ribbon (which relates to bonding of the radical edges to the substrate ${ }^{21}$ ) and of the distinct maxima related to $\mathrm{H}_{2}$ edge termination (see Supplementary Fig. 2), respectively. Thus, our 6-ZGNRs exhibit atomically precise edges with the expected $\mathrm{CH}$ termination. Both features are crucial to host the predicted antiferromagnetic edge states.

As can be seen from the STM image in Fig. 2b, the chemistry of the ZGNR fabrication process faces intrinsic complications such as frequent thermally induced chemical cross-linking of ribbons during 
a

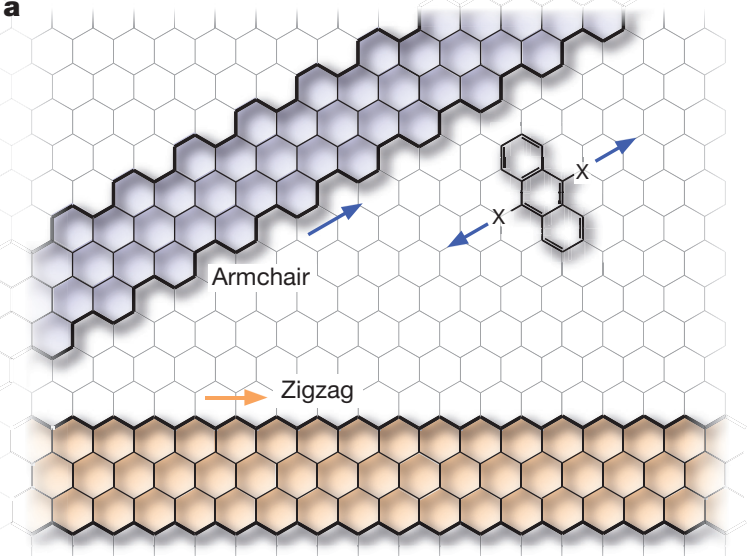

b
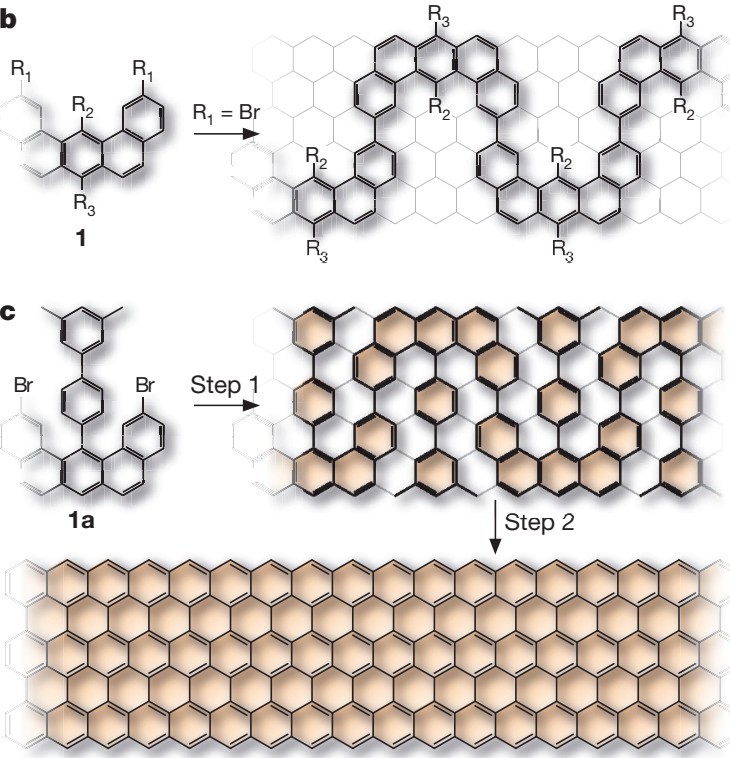

surface-assisted aryl-aryl coupling into a snake-like polymer along the zigzag direction. c, Monomer 1a, with an additional dimethyl-biphenyl group in the interior of the $U$-shape ( $R_{2}$ position), which is designed to afford a 6-ZGNR upon polymerization (step 1 ) and subsequent cyclization (step 2).

level, with energy splittings of $\Delta^{0}=1.5 \mathrm{eV}$ and $\Delta^{1}=1.9 \mathrm{eV}$ between the two occupied states and the unoccupied one (Fig. 3b). $\mathrm{d} I / \mathrm{d} V$ maps acquired at these peaks demonstrate that the corresponding states are highly localized at the zigzag edges (Fig. 3d). Their characteristic features, such as a protrusion at each outermost zigzag carbon atom and an enhanced intensity at the ribbon terminus, are in excellent agreement with the local density of states (DOS) of the corresponding Kohn-Sham density functional theory (DFT) orbitals shown in Fig. 3e. Although effective mean-field theories, such as Kohn-Sham DFT, tend to provide reliable information about the energy level ordering and the shape of orbitals in graphene nanostructures, the same is not true for the size of the electronic gap. More accurate predictions of the bandgaps of graphene nanoribbons are obtained using the $G W$ approximation of many-body perturbation theory ${ }^{23}$ (where ' $G$ ' and ' $W$ ' stand for 'Green's function' and 'screened interaction', respectively).
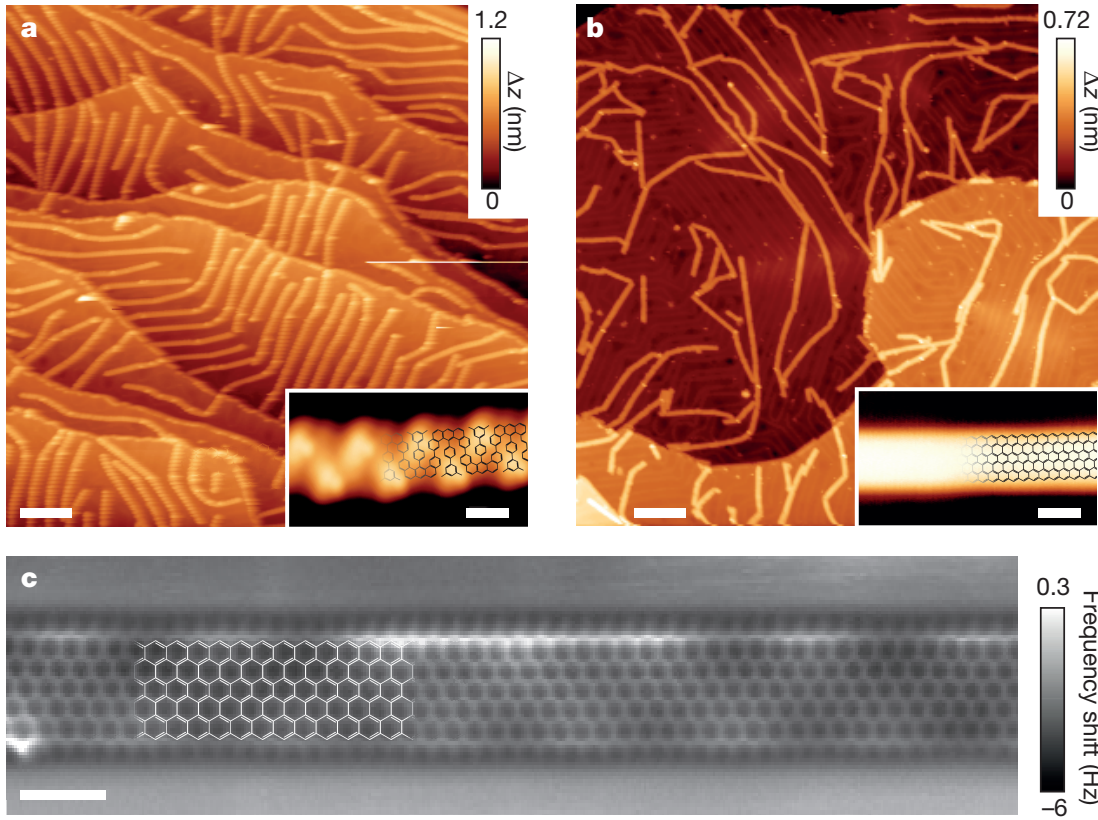

Figure $2 \mid$ Synthesis and characterization of atomically precise 6-ZGNRs. a, Large-scale STM image of the $\mathrm{Au}(111)$ surface after deposition of monomer 1a on the surface held at $475 \mathrm{~K}$. Formation of snake-like polymers is observed. Scanning parameters: voltage $V=-1.5 \mathrm{~V}$, current $I=40 \mathrm{pA}$, colour-scale range $\Delta z=1.2 \mathrm{~nm}$. Scale bar, $20 \mathrm{~nm}$. Inset, high-resolution STM image of the polymer. Zigzag alternation of bright maxima indicates the lifting and/or tilting of the phenyl rings carrying the methyl groups. A structural model is superimposed for comparison. $V=-1.3 \mathrm{~V}, I=10 \mathrm{pA}, \Delta z=0.3 \mathrm{~nm}$. Scale bar, $1 \mathrm{~nm}$. b, Large-scale STM image of the $\mathrm{Au}(111)$ surface after annealing at $625 \mathrm{~K}$. The flatter appearance, reduced apparent height and lack of internal structure indicate the complete cyclodehydrogenation of the polymers and the formation of 6-ZGNRs. $V=-1.0 \mathrm{~V}, I=20 \mathrm{pA}$, $\Delta z=0.7 \mathrm{~nm}$. Scale bar, $20 \mathrm{~nm}$. Inset, high-resolution STM of a 6-ZGNR, which is in excellent agreement with the superimposed structural model. $V=-0.3 \mathrm{~V}, I=5 \mathrm{pA}$, $\Delta z=0.2 \mathrm{~nm}$. Scale bar, $1 \mathrm{~nm}$. c, Constant height nc-AFM frequency shift image taken with a $\mathrm{CO}$-functionalized tip. The intra-ribbon resolution shows the formation of a 6-ZGNR with atomically precise $\mathrm{CH}$ edges. $\mathrm{A} \mathrm{CH}_{2}$ defect is seen in the lower left corner. Oscillation amplitude $A_{\text {osc }}=0.7 \AA ̊ ., V=5 \mathrm{mV}$. Scale bar, $1 \mathrm{~nm}$. 

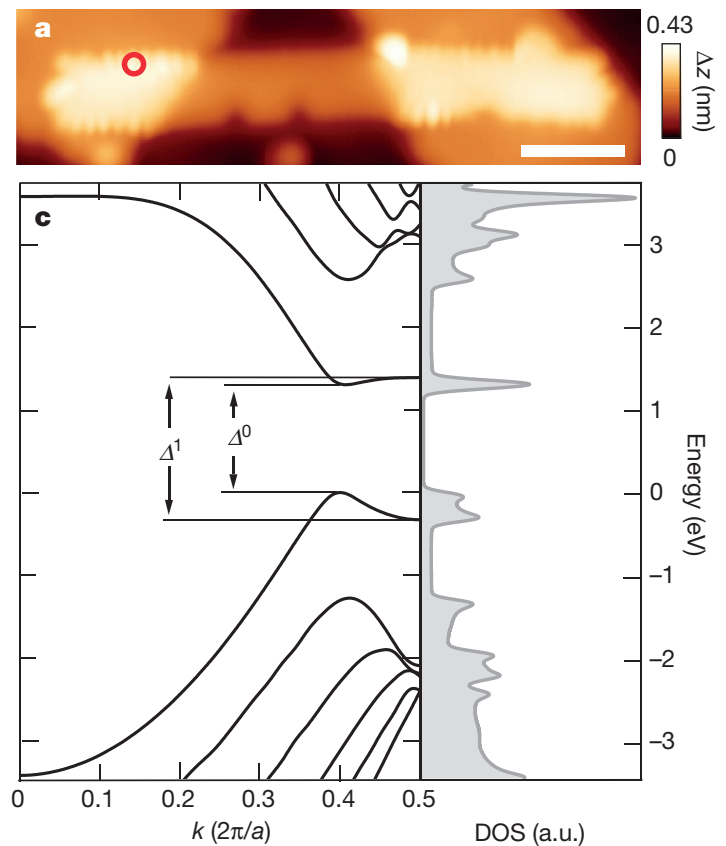

Figure 3 | Edge-state characterization of 6-ZGNRs. a, STM topography image $(V=-0.25 \mathrm{~V}, I=100 \mathrm{pA})$ of a 6-ZGNR bridging between two $\mathrm{NaCl}$ monolayer islands, achieved through STM manipulation. Scale bar, $2 \mathrm{~nm}$. b, Differential conductance ( $\mathrm{d} I / \mathrm{d} V)$ spectrum (red) taken at the zigzag edge marked by the red circle in a and the quasiparticle density of states (DOS; grey). c, Quasiparticle band structure (energy versus wave vector k) (left, black; $a$ is the lattice parameter) and DOS (right, grey) calculated

The resultant quasiparticle band structure and the corresponding DOS are presented in Fig. $3 \mathrm{c}$. The calculated energy splittings $\left(\Delta^{0}=1.4 \mathrm{eV}\right.$ and $\Delta^{1}=1.7 \mathrm{eV}$ ) are in good agreement with experiment. (The quantitative agreement between the experimentally obtained energy splittings and those calculated using many-body perturbation theory is attributed to a compensation of bandgap reduction due to screening by the underlying metal and $\mathrm{NaCl}$ island, and to bandgap increase due to

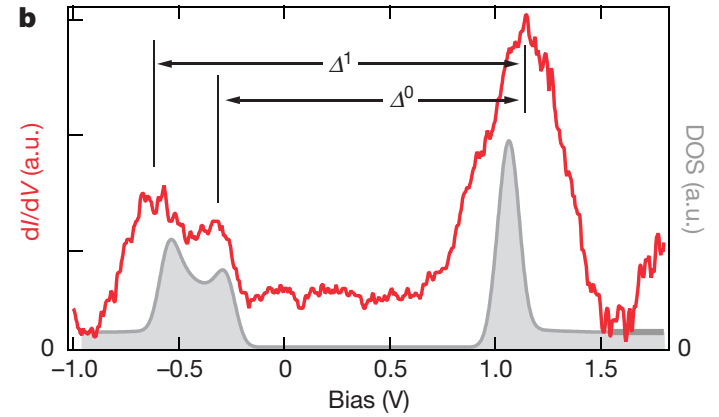

d
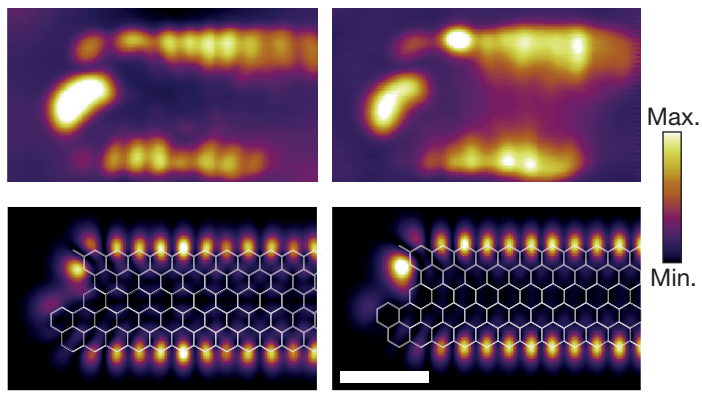

for an infinitely long 6-ZGNR. d, Differential-conductance maps of filled (left) and empty (right) edge states taken at a sample bias of $-0.3 \mathrm{~V}$ and $1.0 \mathrm{~V}$, respectively. e, DFT-based local DOS at a 4 - $\AA$ tip-sample distance, showing the spatial distribution of filled (left, with overlaid structural model) and empty (right, with overlaid structural model) edge states. Scale bar for $\mathbf{d}$ and $\mathbf{e}, 1 \mathrm{~nm}$. a.u., arbitrary units.

quantum confinement within the finite decoupled ribbon segments.) This agreement, in conjunction with the agreement between experimentally determined and simulated DOS maps, clearly evidences the experimental observation of the unperturbed zigzag edge states. Although edge states have previously been observed in several systems with less-well-defined zigzag edges ${ }^{9,24-27}$, the reported energy splittings vary greatly and are substantially smaller than those reported a
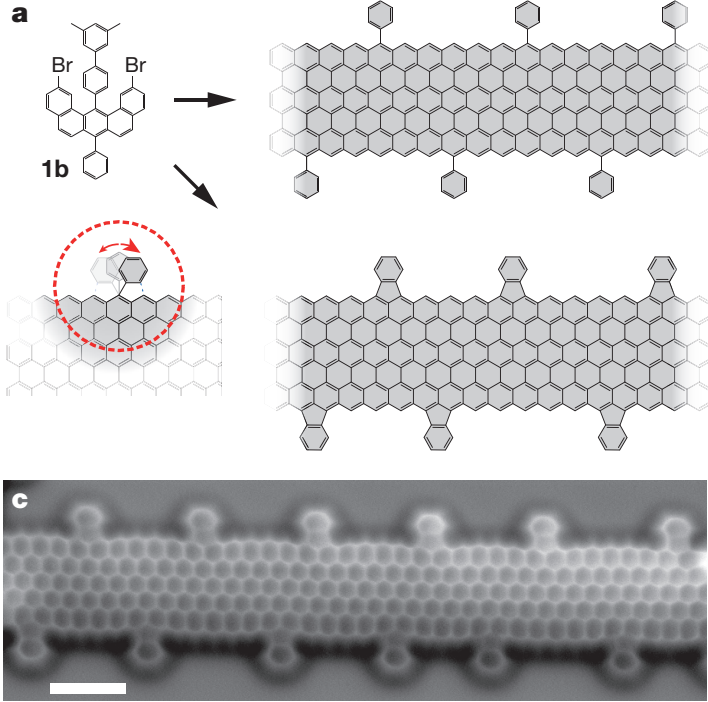

Figure 4 | Synthesis and characterization of edge-modified 6-ZGNRs. a, Monomer $\mathbf{1 b}$ (top left) with an additional phenyl group at the $\mathrm{R}_{3}$ position of the monomer 1a, which is designed to afford an edgemodified 6-ZGNR upon polymerization and subsequent cyclization. The highlighted scheme (dashed red circle) illustrates the two possible rotation possibilities (bottom left, red arrow) upon activation of the external phenyl ring. Right, possible cyclodehydrogenation products assuming no activation of the external phenyl groups (top) and formation of

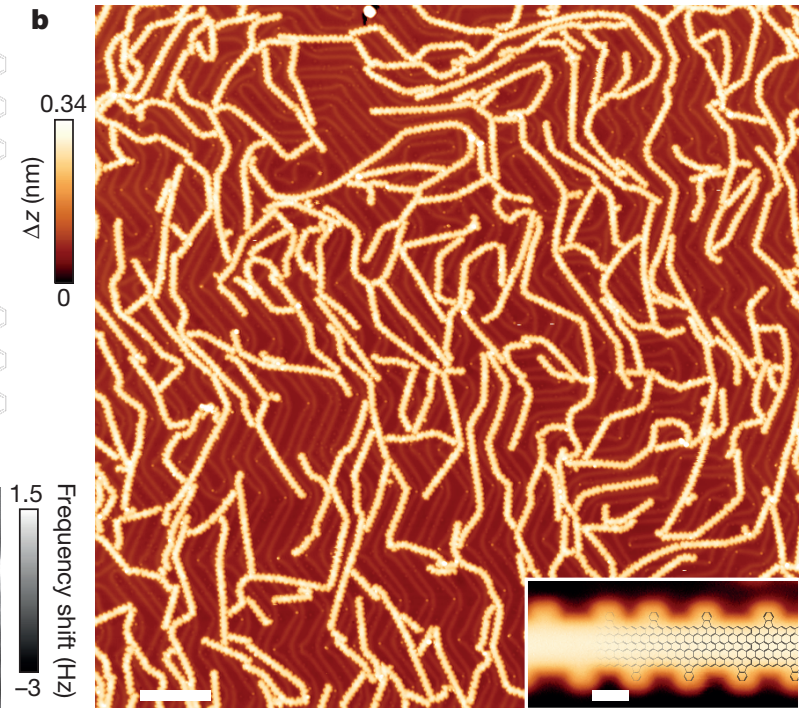

fluoranthene subunits based on an additional dehydrogenative ring closure at the external phenyl groups (bottom). b, Overview STM image ( $V=-1.5 \mathrm{~V}, I=150 \mathrm{pA}$ ) of edge-modified 6-ZGNRs fabricated on a $\mathrm{Au}(111)$ surface. Scale bar, $20 \mathrm{~nm}$. Inset, high-resolution STM image $(V=0.15 \mathrm{~V}, I=2 \mathrm{pA})$. Scale bar, $1 \mathrm{~nm}$. c, Constant-height nc-AFM frequency-shift image of edge-modified 6-ZGNR $\left(A_{\text {osc }}=0.7 \AA\right.$, $V=25 \mathrm{mV})$. Scale bar, $1 \mathrm{~nm}$. 
here. This discrepancy indicates that the electronic structure of zigzag edges is extremely sensitive to edge roughness and interaction with the supporting substrate.

The synthesis of GNRs with perfect zigzag edge periphery combined with convincing evidence of their edge states could lead to new methods for experimentally verifying the electronic, optical and magnetic properties predicted by theory ${ }^{28-30}$, and to the systematic engineering of these properties by using modified types of ZGNRs. As a first step along these lines, we further refined our monomer design by introducing an analogous compound $\mathbf{1 b}$ (Fig. 4), which is similar to $\mathbf{1 a}$ except that it bears an additional phenyl group at the $\mathrm{R}_{3}$ position (see Supplementary Information). We expected that, owing to the steric hindrance brought about by the twisted phenyl group, the growing ribbons would be more efficiently decoupled from the surface and potentially better shielded from neighbouring GNRs. The structural characterization of the ZGNRs obtained from monomer $\mathbf{1} \mathbf{b}$ in an analogous, thermally induced polymerization-cyclization procedure is shown in Fig. 4b, c. The nc-AFM images clearly reveal that, at the cyclodehydrogenation temperature of $573 \mathrm{~K}$, the external phenyl group undergoes a ring closure under the formation of a fluoranthene-type subunit with an incorporated five-membered ring, as illustrated in Fig. 4a. Because the additional ring closure can occur by dehydrogenation of either of the neighbouring zigzag-edge carbon atoms, we do not expect to observe fully periodic arrangement of the fluoranthene subunits. This expectation is confirmed by the nc-AFM image shown in Fig. 4c, which reveals three, four and five zigzag cusps that separate neighbouring fluoranthene subunits.

Controlled edge modification is a useful strategy for engineering the band structure of edge states. However, the emphasis here is on the reduction of ZGNR-substrate interaction. Indeed, the edge modification discussed above alters the ZGNR-substrate interaction enough to enable the STM to map the typical features of the edge state (see Supplementary Fig. 7).

The successful bottom-up synthesis of atomically precise ZGNRs opens extensive opportunities and challenges for the analysis of their physical properties (for example, their band structure, magnetism and charge/spin transport) and for the fabrication of ZGNR-based devices such as the proposed spin valves ${ }^{12}$. However, the strong interaction of the pristine 6-ZGNR with the metal substrate (as reflected in the obstruction of the spectral features of the edge states) raises important questions regarding the chemical reactivity of the zigzag edges, which needs to be controlled to be able to study and apply these materials under ambient conditions. A promising method for doing so is provided by edge 'passivation' approaches that preserve the electronic properties of the ribbon, but reduce the chemical reactivity of their (functionalized) edges ${ }^{31}$.

Online Content Methods, along with any additional Extended Data display items and Source Data, are available in the online version of the paper; references unique to these sections appear only in the online paper.

\section{Received 7 July 2015; accepted 19 January 2016.}

1. Saito, R., Fujita, M., Dresselhaus, G. \& Dresselhaus, M. S. Electronic structure of graphene tubules based on $\mathrm{C}_{60}$. Phys. Rev. B 46, 1804-1811 (1992).

2. Wakabayashi, K., Fujita, M., Ajiki, H. \& Sigrist, M. Electronic and magnetic properties of nanographite ribbons. Phys. Rev. B 59, 8271-8282 (1999).

3. Han, W., Kawakami, R. K., Gmitra, M. \& Fabian, J. Graphene spintronics. Nature Nanotechnol. 9, 794-807 (2014)

4. Nakada, K., Fujita, M., Dresselhaus, G. \& Dresselhaus, M. S. Edge state in graphene ribbons: nanometer size effect and edge shape dependence. Phys. Rev. B 54, 17954-17961 (1996).

5. Han, M. Y., Ozyilmaz, B., Zhang, Y. \& Kim, P. Energy band-gap engineering of graphene nanoribbons. Phys. Rev. Lett. 98, 206805 (2007).

6. Kosynkin, D. V. et al. Longitudinal unzipping of carbon nanotubes to form graphene nanoribbons. Nature 458, 872-876 (2009).
7. Li, X., Wang, X., Zhang, L., Lee, S. \& Dai, H. Chemically derived, ultrasmooth graphene nanoribbon semiconductors. Science 319, 1229-1232 (2008).

8. Wang, X. \& Dai, H. Etching and narrowing of graphene from the edges. Nature Chem. 2, 661-665 (2010).

9. Magda, G. Z. et al. Room-temperature magnetic order on zigzag edges of narrow graphene nanoribbons. Nature 514, 608-611 (2014).

10. Ma, L., Wang, J. \& Ding, F. Recent progress and challenges in graphene nanoribbon synthesis. ChemPhysChem 14, 47-54 (2013).

11. Topsakal, M., Sevincli, H. \& Ciraci, S. Spin confinement in the superlattices of graphene ribbons. Appl. Phys. Lett. 92, 173118 (2008).

12. Wimmer, M., Adagideli, i., Berber, S., Tománek, D. \& Richter, K. Spin currents in rough graphene nanoribbons: universal fluctuations and spin injection. Phys. Rev. Lett. 100, 177207 (2008).

13. Son, Y.-W., Cohen, M. L. \& Louie, S. G. Half-metallic graphene nanoribbons. Nature 444, 347-349 (2006)

14. Cai, J. et al. Atomically precise bottom-up fabrication of graphene nanoribbons. Nature 466, 470-473 (2010).

15. Chen, Y.-C. et al. Tuning the band gap of graphene nanoribbons synthesized from molecular precursors. ACS Nano 7, 6123-6128 (2013).

16. Zhang, H. et al. On-surface synthesis of rylene-type graphene nanoribbons. J. Am. Chem. Soc. 137, 4022-4025 (2015).

17. Bronner, C. et al. Aligning the band gap of graphene nanoribbons by monomer doping. Angew. Chem. Int. Ed. 52, 4422-4425 (2013).

18. Cai, J. et al. Graphene nanoribbon heterojunctions. Nature Nanotechnol. 9, 896-900 (2014)

19. Chen, Y.-C. et al. Molecular bandgap engineering of bottom-up synthesized graphene nanoribbon heterojunctions. Nature Nanotechnol. 10, 156-160 (2015).

20. Gross, L., Mohn, F., Moll, N., Liljeroth, P. \& Meyer, G. The chemical structure of a molecule resolved by atomic force microscopy. Science 325, 1110-1114 (2009).

21. Li, Y., Zhang, W., Morgenstern, M. \& Mazzarello, R. Electronic and magnetic properties of zigzag graphene nanoribbons on the (111) surface of $\mathrm{Cu}, \mathrm{Ag}$, and Au. Phys. Rev. Lett. 110, 216804 (2013).

22. Repp, J., Meyer, G., Stojković, S. M., Gourdon, A. \& Joachim, C. Molecules on insulating films: scanning-tunneling microscopy imaging of individual molecular orbitals. Phys. Rev. Lett. 94, 026803 (2005)

23. Yang, L., Park, C.-H., Son, Y.-W., Cohen, M. L. \& Louie, S. G. Quasiparticle energies and band gaps in graphene nanoribbons. Phys. Rev. Lett. 99, 186801 (2007).

24. Ritter, K. A. \& Lyding, J. W. The influence of edge structure on the electronic properties of graphene quantum dots and nanoribbons. Nature Mater. $\mathbf{8}$, 235-242 (2009)

25. Tao, C. et al. Spatially resolving edge states of chiral graphene nanoribbons. Nature Phys. 7, 616-620 (2011).

26. van der Lit, J. et al. Suppression of electron-vibron coupling in graphene nanoribbons contacted via a single atom. Nature Commun. 4, 2023 (2013).

27. Li, Y. Y., Chen, M. X., Weinert, M. \& Li, L. Direct experimental determination of onset of electron-electron interactions in gap opening of zigzag graphene nanoribbons. Nature Commun. 5, 4311 (2014).

28. Dutta, S. \& Wakabayashi, K. Tuning charge and spin excitations in zigzag edge nanographene ribbons. Sci. Rep. 2, 519 (2012).

29. Yang, L., Cohen, M. L. \& Louie, S. G. Magnetic edge-state excitons in zigzag graphene nanoribbons. Phys. Rev. Lett. 101, 186401 (2008)

30. Yazyev, O. V. A guide to the design of electronic properties of graphene nanoribbons. Acc. Chem. Res. 46, 2319-2328 (2013).

31. Li, Y., Zhou, Z., Cabrera, C. R. \& Chen, Z. Preserving the edge magnetism of zigzag graphene nanoribbons by ethylene termination: insight by Clar's rule. Sci. Rep. 3, 2030 (2013).

Supplementary Information is available in the online version of the paper.

Acknowledgements This work was supported by the Swiss National Science Foundation, the Office of Naval Research BRC Program, the European Research Council (grant NANOGRAPH), the DFG Priority Program SPP 1459, the Graphene Flagship (No. CNECT-ICT-604391), and the European Union Projects UPGRADE, GENIUS and MoQuaS. We acknowledge the Swiss Supercomputing Center (CSCS) for computational resources (project s507). We thank A. Ferretti for his contribution to this project and O. Gröning for discussions.

Author Contributions P.R., R.F., X.F. and K.M. conceived and supervised the experiments. B.Y. and T.Du. synthesized the precursor monomers. J.L. and C.S developed the on-surface synthesis protocols and did the STM analysis. T.Di., J.L. and S.W. performed the AFM imaging; S.W. and J.L. did the spectroscopic analysis. P.S., L.T., C.A.P. and D.P. performed the simulations. C.S., S.W. and P.R. made the figures. P.R., K.M. and R.F. wrote the paper. All authors discussed the results and implications and commented on the manuscript at all stages.

Author Information Reprints and permissions information is available at www.nature.com/reprints. The authors declare no competing financial interests. Readers are welcome to comment on the online version of the paper. Correspondence and requests for materials should be addressed to R.F. (roman.fasel@empa.ch) or K.M. (muellen@mpip-mainz.mpg.de). 


\section{METHODS}

Sample preparation. Experiments were carried out under ultra-high vacuum conditions (base pressure $10^{-11} \mathrm{mbar}$ ) with a low-temperature STM and a lowtemperature STM/AFM, both from Omicron-Oxford. A Au(111) single crystal was used as the substrate for the growth of 6-ZGNRs and edge-modified 6-ZGNRs. The $\mathrm{Au}(111)$ surface was cleaned by repeated cycles of argon-ion bombardment and annealing at $750 \mathrm{~K}$ for $15 \mathrm{~min}$ until a clean surface was obtained, as judged by STM. Molecular precursors were thermally deposited on the clean $\mathrm{Au}(111)$ surface held at room temperature with a typical rate of $1 \AA \mathrm{min}^{-1}$. After deposition, the sample was post-annealed in four steps to $475 \mathrm{~K}, 525 \mathrm{~K}, 575 \mathrm{~K}$ and $625 \mathrm{~K}$ for typically 15 min per step to achieve long, high-quality GNRs. A modified growth protocol was used for the experiments in which 6-ZGNRs are transferred onto $\mathrm{NaCl}$ islands. After precursor deposition at room temperature, the substrate temperature was directly increased to $625 \mathrm{~K}\left(1 \mathrm{~K} \mathrm{~s}^{-1}\right)$ and kept at this temperature for $5 \mathrm{~min}$. This protocol increases the number of short and isolated 6-ZGNRs needed for successful manipulation with the STM tip. Compared to the protocol described above, this increases the number of defects at the ZGNR edges.

Imaging, manipulation and spectroscopy.

Constant-current STM imaging. STM images were acquired in the constant-current mode at sample temperatures of $77 \mathrm{~K}$ or $5 \mathrm{~K}$, as indicated in each case. Scanning parameters are specified in each figure caption.

Constant-height, non-contact AFM imaging. Non-contact AFM measurements were performed with a tungsten tip attached to a tuning fork sensor ${ }^{32}$. The tip was a posteriori functionalized by the controlled adsorption of a single $\mathrm{CO}$ molecule at the tip apex from the previously CO-dosed surface ${ }^{33}$. This procedure enables the imaging of the chemical structure of organic molecules ${ }^{20}$. The sensor was driven close to its resonance frequency (about $23,570 \mathrm{~Hz}$ ) with a constant amplitude of approximately $70 \mathrm{pm}$. The shift in the resonance frequency of the tuning fork (with the attached CO-functionalized tip) was recorded in constant-height mode (Omicron Matrix electronics and HF2Li PLL by Zurich Instruments).

Transfer of GNRs onto $\mathrm{NaCl}$ monolayer islands. We developed a routine to transfer bottom-up fabricated short GNRs from the metal surface onto insulating $\mathrm{NaCl}$ islands in order to be able to access their intrinsic electronic structure. This method, in which physisorbed individual 6-ZGNRs are laterally and/or vertically manipulated on the $\mathrm{Au}(111)$ surface, consists of four steps: (1) deposition of $\mathrm{NaCl}$ islands (thermal evaporation of submonolayer coverage, deposition temperature of about $1,000 \mathrm{~K}$ ) on the 6 -ZGNR/Au(111) surface; (2) pick-up of one end of a GNR by approaching and retracting the STM tip with low bias (about $-50 \mathrm{mV}$ ) (3) lateral displacement of the tip, together with the GNR, above the $\mathrm{NaCl}$ island; and (4) release of the ribbon by a $3.0 \mathrm{~V}$ voltage pulse, leaving the GNR partially adsorbed on $\mathrm{NaCl}$ and partially on the metal surface.

Differential-conductance spectroscopy. The differential conductance $(\mathrm{d} I / \mathrm{d} V)$ measurements were performed in a low-temperature STM at $5 \mathrm{~K}$ via the lock-in technique, using a bias-voltage modulation of $20 \mathrm{mV}$ and a frequency of $860 \mathrm{~Hz}$. $\mathrm{d} / \mathrm{d} V$ maps were acquired in the constant-current mode for the decoupled 6-ZGNR, and in the constant-height mode for ribbons on $\mathrm{Au}(111)$.
Theoretical details. DFT calculations were carried out using the CP2K code (http://www.cp2k.org) for geometry optimizations; the Quantum ESPRESSO code $^{34}$ was used for scanning tunnelling spectroscopy simulations. In CP2K, the core electrons and nuclei are represented using the GTH pseudopotential ${ }^{35}$ and the valence electrons are treated with a triple- $\zeta$ valence basis set with two sets of $p$-type or $d$-type polarization functions (TZV2P) ${ }^{36}$; in Quantum ESPRESSO, a plane-wave basis set and norm-conserving pseudopotentials are used. The exchange-correlation was approximated by the PBE functional ${ }^{37}$.

The edge-modified 6-ZGNR structures shown in Fig. 4 were set up starting from the optimized atomic structure of the 6-ZGNR (lattice parameter $a=2.461 \AA$ ) and contain 24 unit cells. A $19-\AA$-wide (15- $\AA$-wide) region of vacuum was included along the transverse (perpendicular) directions to avoid interactions between periodic replicas. Forces on the nuclei were then relaxed until all forces dropped below $5 \mathrm{meV} \AA^{-1}$. Using the optimized atomic structure, a self-consistent field calculation was carried out in Quantum ESPRESSO, using energy cut-offs of 120 Ry and 480 Ry $(1 \mathrm{Ry} \approx 13.6 \mathrm{eV})$ for the wave function and the charge density, respectively, and a $k$-point grid of $3 \times 1 \times 1$, including the $\Gamma$-point.

Quasi-particle corrections were computed within the framework of many-body perturbation theory, using a single iteration $\left(G_{0} W_{0}\right)$ in the $G W$ approximation to the self-energy as implemented in the yambo code ${ }^{38}$. The electronic structure of the 6-ZGNRs from DFT was recalculated using a 60 -Ry plane-wave cut-off, $64 k$-points in the first Brillouin zone and 250 bands, covering the energy range up to $21 \mathrm{eV}$ above the highest occupied band. The dielectric matrix $(\varepsilon)$ was calculated in the random phase approximation with an 8-Ry cut-off for the plane-wave basis. $\varepsilon^{-1}$ was evaluated at frequencies of $\omega=0$ and $\omega=i 2$ Ry and extended to the real frequency axis using the plasmon-pole model by ref. 39. A rectangular Coulomb cut-off was used along the directions perpendicular to the GNR axis, as described in ref. 40.

32. Giessibl, F. J. Atomic resolution on Si(111)-(7 $\times 7)$ by noncontact atomic force microscopy with a force sensor based on a quartz tuning fork. Appl. Phys. Lett. 76, 1470-1472 (2000).

33. Bartels, L. et al. Dynamics of electron-induced manipulation of individual CO molecules on Cu(111). Phys. Rev. Lett. 80, 2004-2007 (1998).

34. Giannozzi, P. et al. QUANTUM ESPRESSO: a modular and open-source software project for quantum simulations of materials. J. Phys. Condens. Matter 21, 395502 (2009)

35. Goedecker, S., Teter, M. \& Hutter, J. Separable dual-space Gaussian pseudopotentials. Phys. Rev. B 54, 1703-1710 (1996)

36. VandeVondele, J. \& Hutter, J. Gaussian basis sets for accurate calculations on molecular systems in gas and condensed phases. J. Chem. Phys. 127, 114105 (2007).

37. Perdew, J. P., Burke, K. \& Ernzerhof, M. Generalized gradient approximation made simple. Phys. Rev. Lett. 77, 3865 (1996).

38. Marini, A., Hogan, C., Grüning, M. \& Varsano, D. yambo: an ab initio tool for excited state calculations. Comput. Phys. Commun. 180, 1392-1403 (2009).

39. Godby, R. W. \& Needs, R. J. Metal-insulator transition in Kohn-Sham theory and quasiparticle theory. Phys. Rev. Lett. 62, 1169-1172 (1989).

40. Rozzi, C. A. Varsano, D., Marini, A. Gross, E. K. U. \& Rubio, A. Exact Coulomb cutoff technique for supercell calculations. Phys. Rev. B 73, 205119 (2006). 